\title{
Relationship between Insulin Sensitivity and Muscle Lipids may Differ with Muscle Group and Ethnicity
}

\author{
Jeannine C. Lawrence ${ }^{*}, 1,2$, Barbara A. Gower ${ }^{2}$, W. Timothy Garvey², A. Julian Muñoz ${ }^{2}$, \\ Betty E. Darnell ${ }^{3}$, Robert A. Oster ${ }^{3}$, Steven D. Buchthal ${ }^{4}$, Michael I. Goran ${ }^{5}$ and \\ Bradley R. Newcomer ${ }^{4}$
}

${ }^{I}$ Department of Human Nutrition, University of Alabama, Tuscaloosa, Alabama, USA

${ }^{2}$ Department of Nutrition Sciences and Clinical Nutrition Research Center, University of Alabama at Birmingham, Birmingham, Alabama, USA

${ }^{3}$ The Pittman General Clinical Research Center, University of Alabama at Birmingham, Birmingham, Alabama, USA

${ }^{4}$ Department of Diagnostic and Therapeutic Sciences, University of Alabama at Birmingham, Birmingham, Alabama, USA

${ }^{5}$ Departments of Preventive Medicine, and Department of Physiology and Biophysics, University of Southern California, Los Angeles, CA, USA

\begin{abstract}
Intramyocellular lipid (IMCL) has been inversely associated with insulin sensitivity in some, but not all, studies. This study utilized fast, high-resolution, magnetic resonance spectroscopic imaging (MRSI) to: investigate relationships between muscle lipids (IMCL and extramyocellular lipid (EMCL)) and insulin sensitivity in muscles of varying oxidative capacity, explore ethnic differences in these relationships, and determine whether a eucaloric, low-fat dietary intervention would reduce IMCL and increase insulin sensitivity. Subjects were 30 healthy, African-American $(A A ; n=14)$ and European-American $(E A ; n=16)$ males, BMI $26.49( \pm 5.57) \mathrm{kg} / \mathrm{m}^{2}$, age $21.80( \pm 7.84)$ yrs. Soleus and tibialis anterior muscle lipids were quantified using MRSI. Insulin sensitivity was assessed via intravenous glucose tolerance test. A 2-week, eucaloric, low-fat diet intervention was conducted in a sub-group ( $\mathrm{n}=12$ ) subjects with assessments at baseline and post-intervention. Neither IMCL nor EMCL levels differed between ethnicities. In the total group, and within EA (but not AA), both tibialis anterior IMCL and EMCL were inversely associated with insulin sensitivity $(P<0.05$ for both); soleus muscle lipids were not associated with insulin sensitivity. Soleus, but not tibialis anterior, IMCL declined in both ethnic groups (average $25.3 \% ; \mathrm{p}<0.01$ ) following dietary intervention; insulin sensitivity was unchanged. Results suggest that an association of muscle lipids with insulin sensitivity may be influenced by the oxidative capacity of the muscle group studied and may vary with ethnicity.
\end{abstract}

Keywords: Muscle lipids, intramyocellular lipid, MRSI, ethnicity, insulin sensitivity.

\section{INTRODUCTION}

The role of excess muscle lipid accumulation (as intramyocellular (IMCL) and extramyocellular lipid (EMCL)) and its potentially detrimental effect on insulin sensitivity has been under active investigation by many scientific groups [1-5]. This negative association has been proposed to be causal, a hypothesis that is supported by data linking fatty acid metabolites to defects in insulin signaling [6-8]. However, the cause-and-effect nature of the relationship has not been confirmed. The absence of an association between IMCL and insulin sensitivity among normoglycemic women [9] suggests that the association may be more readily apparent in metabolically perturbed or "at risk" subjects, such as those predisposed to or with type 2 diabetes, or the elderly

*Address correspondence to this author at the University of Alabama, Department of Human Nutrition and Hospitality Mgt., P.O. Box 870158, Tuscaloosa, AL 35487-0158, USA; Tel: (205) 348-6252; Fax: (205) 3482982; E-mail: jlawrence@ches.ua.edu
[3-5]. This population-specific association calls into question whether IMCL is a direct contributor to decreased insulin sensitivity, or if, instead, it is either a marker for metabolic state, or the result of a metabolic perturbation [10]. Therefore, it is important to examine the association between IMCL and insulin sensitivity in healthy, free-living populations, and to determine if manipulation of IMCL is associated with changes in insulin sensitivity.

One potential cause for the discrepancies among studies is the specific muscles investigated. The soleus muscle is commonly used because it is the largest muscle in the calf and is oriented parallel to the magnetic field during magnetic studies. These characteristics allow for easy identification and collection of spectra with increased spectral quality. However, the soleus muscle is a highly oxidative muscle, displaying significantly higher succinic dehydrogenase activity as compared to the adjacent tibialis anterior muscle [11]. Such biochemical differences in oxidative activity between muscle groups may affect both muscle lipid utilization and 
the relationship of muscle lipid content to whole-body insulin sensitivity $[12,13]$. Therefore, studies assessing the association between muscle lipid content and insulin sensitivity should assess several muscles that differ in oxidative activity.

Ethnicity is known to be a profound determinant of insulin sensitivity. African Americans (AA), vs. European Americans (EA), are less insulin sensitive independent of obesity and body fat distribution [14-17]. Several studies have reported greater total muscle lipid in AA vs. EA [1821]. A study of $60 \mathrm{AA}$ and EA women found that higher intermuscular adipose tissue independently predicted lower insulin sensitivity [20]. In that study, the authors recommended future studies incorporate direct assessment of IMCL and EMCL to clarify the associations of these depots with insulin sensitivity. An editorial accompanying this publication suggested that future research should investigate ethnic differences in the relationship of muscle lipids to metabolic outcomes, such as insulin sensitivity, using a closely matched cohort [22].

Our laboratory recently developed a method of assessing muscle lipids using a novel, fast, two-dimensional magnetic resonance spectroscopic imaging (MRSI) sequence [23]. This method involves utilizing a short repetition time $(\mathrm{TR}=$ $145.9 \mathrm{~ms}$ ) 2D MRSI sequence to achieve a very high resolution data set (volume-per-voxel $=0.0625 \mathrm{cc}$ ) in a brief period of time ( $\sim 10$ minutes). Overall, this new sequence greatly increased voxel spatial resolution, allowed for simultaneous assessment of multiple muscle groups, and significantly decreased the total scan time and subject burden [23]. This methodology may be valuable for studies requiring rapid or repeated assessment of muscle lipid. However, the application of this methodology to physiologic studies involving metabolic outcomes has not been examined.

This study was conducted in a group of healthy, freeliving $\mathrm{AA}$ and $\mathrm{EA}$ males to investigate the relationship between muscle lipids (as IMCL and EMCL) and insulin sensitivity in muscles varying in oxidative activity. The study used our newly developed, fast, high-resolution, MRSI technique, which allowed us to investigate multiple muscle groups with differing levels of oxidative activity and lipid levels in a single, short-duration, acquisition. Secondary aims of this study were to determine whether ethnic differences existed in the association between muscle lipids and insulin sensitivity, and whether manipulation of muscle lipids via low-fat diet both reduced IMCL and increased insulin sensitivity.

\section{MATERIALS AND METHODOLOGY}

\section{Subjects}

Subjects were 30 free-living, adult males, 14 of AA and 16 of EA descent recruited from the University community and the surrounding Birmingham area. Subjects were enrolled in one of two studies, which were approved by the Institutional Review Board at the University of Alabama at Birmingham. Both subjects, and the parents of any subjects who were minors, provided informed consent. All subjects were considered adult based on pubertal stage of 5 , according to the criteria of Marshall and Tanner [24]. Subjects were excluded if they were taking medications, diagnosed with syndromes known to affect body composition or physical activity, or had experienced chronic, recurrent infections or any major illnesses. Additional criteria for inclusion in the dietary intervention sub-study $(n=12)$ were: aged $19-45$ years, body mass index [BMI, defined as weight in $\mathrm{kg} / \mathrm{height}$ in $\left(\mathrm{m}^{2}\right)$ ] of 24.9 to 33.0 , healthy, nonsmokers, and no history of diabetes, cardiovascular disease, hypertension, or chronic illness. Exclusion criteria for this study included having any metal objects in their body or participating in habitual moderate or aerobic exercise totaling more than 2 hours per week.

\section{Protocol}

Subjects reported to the UAB Center for Nuclear Imaging Research (CNIR) to undergo MRSI. They were then admitted in the late afternoon to the General Clinical Research Center (GCRC) for an overnight stay. At approximately $1800 \mathrm{~h}$, subjects were provided a standardized dinner meal consisting of $55 \%$ carbohydrate, $15 \%$ protein, and $30 \%$ fat (total group, baseline) or $\sim 60 \%$ carbohydrate, $\sim 30 \%$ protein, and $<10 \%$ fat (post-diet intervention). A small snack was also provided and was consumed by $2000 \mathrm{~h}$. After $2000 \mathrm{~h}$, only water or noncaffeinated, noncaloric beverages were allowed until after testing was completed the following day. Soft, flexible catheters were placed in each arm the following morning to collect blood for serum analyses and to conduct insulin sensitivity testing. Testing was conducted while subjects were in a fasted condition. Testing was conducted in all subjects $(n=30)$ at baseline and following the 2-week, low-fat diet-intervention arm in a subset of subjects $(n=12)$.

\section{Body Composition}

Total and regional body composition (fat mass and lean mass) were measured by dual-energy X-ray absorptiometry (DXA) using a GE Lunar Prodigy densitometer (GE Lunar Corporation, Madison, WI). All scans were performed and analyzed according to manufacturer's instructions with enCORE 2002 Version 6.10.029 software. Specialized analyses were performed using a "Region-of-Interest" (ROI) "Custom" analysis sequence. For quantification of total leg tissue, a box was manually drawn with the upper boundary of the ROI formed by a line placed through the joint of the femur adjacent to the angle of the pelvis, and the remaining 3 sides placed on either side of the leg and below the foot.

\section{Intramuscular Lipid Measurements}

All measurements were performed on a $4.1 \mathrm{~T}$ whole body imaging and spectroscopy system at the CNIR, which was interfaced to a Bruker console (Bruker Instruments, Inc). All acquisition and processing methods for this study have been previously described [23]. Briefly, intramuscular lipids (IMCL and EMCL) of muscles in the right calf were measured using a slice-selective, fast 2D MRSI sequence. Following the MRSI, a set of high-resolution axial scout images was obtained to co-register with the spectroscopic image so that the muscle group of each pixel could be identified. These anatomical images were collected in 10$\mathrm{mm}$ slices with a slice separation of $15 \mathrm{~mm}$. 
All MRSI data sets were converted and reconstructed using a custom-built software package written in the MatLab version 6.1 (The MathWorks, Inc, Natick, MA). Individual spectra and spectra from regions-of-interest (ROI) were manually chosen and extracted for spectral analysis as previously described and representative spectra from this population have been previously shown by Newcomer et al. [23]. For IMCL determination, a small summed ROI from a $6 \times 6$ set of voxels (soleus) or $4 \times 4$ set of voxels (tibialis anterior) that was free from marbling and produced adequate peak splitting was identified. For EMCL determination, a large summed ROI each for the individual soleus and tibialis anterior muscles was manually drawn and included all voxels inside the facial layers of each of the muscles was identified [23]. These spectra were analyzed by fitting the peak positions and areas through time domain fitting using Java-Based Magnetic Resonance User Interface (jMRUI) version 2.1 [European Consortium of Scientists; 25). IMCL and EMCL content in the soleus spectra were fit using previously published fitting models and sets of prior knowledge information [23, 26-29]. Lipid peak amplitudes were normalized to an external peanut oil phantom using similar methodology as described by Perseghin et al. [30]. Consequently, all peak areas in this study are expressed in arbitrary units (a.u.) relative to the phantom signal amplitude.

\section{Insulin Sensitivity Testing}

At approximately 0700 , a frequently-sampled, intravenous glucose tolerance test (FSIGT) was conducted. Glucose $(300 \mathrm{mg} / \mathrm{kg})$ was administered intravenously at time "zero" followed by insulin $(0.02 \mathrm{U} / \mathrm{kg})$ at time $20 \mathrm{~min}$. Blood samples were collected 24 times ( $72 \mathrm{ml}$ total) in the young adult participants, and 33 times (99 $\mathrm{ml}$ total) in the adults, and were analyzed for glucose and insulin. Glucose and insulin data were entered into Min Mod computer software (version 5.15; Richard N. Bergman, Department of Physiology and Biophysics, University of Southern California, Los Angeles, CA) to generate the insulin sensitivity index, $\mathrm{Si}$ [31].

Glucose concentration was assessed using the glucose oxidase method (Analox, Lunenburg, MA). Insulin was assayed in duplicate $100 \mu 1$ aliquots using double-antibody
RIA with reagents obtained from Linco Research Inc. (St. Charles, MO). The assay sensitivity was $2.9 \mu \mathrm{IU} / \mathrm{ml}$, and mean intra-assay and interassay coefficients of variation were $4.0 \%$ and $3.5 \%$, respectively.

\section{Diet}

Participants in the very low-fat diet-intervention arm of this project $(\mathrm{n}=12)$ were provided with all meals and snacks for 2 weeks by the GCRC Metabolic Kitchen, and instructed to consume only food provided. The test diet was eucaloric, contained an average of 25 grams of fiber/day, and had a macronutrient composition of $<10 \%$ energy from fats, $\sim 60 \%$ from carbohydrates, and the remainder $(\geq 30 \%)$ from protein. Energy needs were calculated using the Harris-Benedict formula multiplied by an activity factor of 1.35 to determine total daily energy requirements, as previously described [32]. All menus were prepared by a registered dietitian using Minnesota Nutrient Data Systems for Research (NDS-R), version 4.06 .

\section{Data Analysis}

Baseline differences in demographic and metabolic outcomes between ethnic groups were assessed with $t$-tests. Pearson's correlation coefficients were calculated to examine relationships between muscle lipids and anthropometric and metabolic variables. Three outliers (defined as $\geq 2$ standard deviations from the mean) were present in muscle lipid data. Data were analyzed as a complete set and with outliers removed. No outcome differences resulted from conducting analyses with $v s$. without outliers, therefore final analyses include all data. Changes in muscle lipids and $\mathrm{Si}$ with the diet intervention were assessed using paired $t$-tests. All statistical tests were two-tailed and were performed using a significance level of 0.05 . Analyses were performed using SAS 9.1 (SAS Institute, Cary, NC).

\section{RESULTS}

\section{Subject Characteristics}

Descriptive characteristics of the total population are summarized in Table $\mathbf{1}$.

Table 1. Descriptive Data and Metabolic Variables; Mean (SD)

\begin{tabular}{|c|c|c|c|c|}
\hline & $\begin{array}{l}\text { Total Group } \\
\quad(\mathbf{N}=\mathbf{3 0})\end{array}$ & $\begin{array}{c}\text { African-American } \\
\qquad(\mathrm{n}=14)\end{array}$ & $\begin{array}{c}\text { European-American } \\
(n=16)\end{array}$ & $P^{\mathrm{a}}$ \\
\hline Age & $21.80(7.84)$ & $21.14(7.29)$ & $22.38(8.50)$ & 0.67 \\
\hline BMI $\left(\mathrm{kg} / \mathrm{m}^{2}\right)$ & $26.49(5.57)$ & $27.64(5.04)$ & $25.48(5.96)$ & 0.30 \\
\hline Total body fat mass $(\mathrm{kg})$ & $21.77(12.40)$ & $21.91(11.46)$ & $21.65(13.55)$ & 0.95 \\
\hline Soleus IMCL (arbitrary units ${ }^{\mathrm{b}}$ ) & $0.98(0.59)$ & $0.93(0.60)$ & $1.03(0.60)$ & 0.67 \\
\hline Soleus EMCL (arbitrary units ${ }^{\mathrm{b}}$ ) & $1.64(0.77)$ & $1.58(0.87)$ & $1.70(0.69)$ & 0.69 \\
\hline Tibialis anterior IMCL (arbitrary units ${ }^{\mathrm{b}}$ ) & $0.48(0.38)$ & $0.58(0.49)^{\mathrm{c}}$ & $0.39(0.26)$ & 0.19 \\
\hline Tibialis anterior EMCL (arbitrary units ${ }^{\mathrm{b}}$ ) & $1.66(1.20)$ & $1.45(1.30)^{\mathrm{c}}$ & $1.82(1.13)$ & 0.42 \\
\hline
\end{tabular}

${ }^{a}$ for difference between ethnic groups, ${ }^{b}$ arbitrary units relative to an external phantom, ${ }^{c} \mathrm{n}=13$. IMCL $=$ intramyocellular lipid; $\mathrm{EMCL}=$ extramyocellular lipid. 
Table 2. Associations between Muscle Lipids and Anthopometric and Serum Data by Ethnicity; r(p)

\begin{tabular}{|c|c|c|c|c|c|c|c|c|}
\hline & \multicolumn{4}{|c|}{$\begin{array}{c}\text { African-American } \\
(\mathrm{n}=14)\end{array}$} & \multicolumn{4}{|c|}{$\begin{array}{c}\text { European-American } \\
(n=16)\end{array}$} \\
\hline & $\begin{array}{l}\text { Soleus } \\
\text { IMCL }\end{array}$ & $\begin{array}{l}\text { Soleus } \\
\text { EMCL }\end{array}$ & $\begin{array}{l}\text { Tibialis anterior } \\
\text { IMCL }\end{array}$ & $\begin{array}{c}\text { Tibialis anterior } \\
\text { EMCL }\end{array}$ & $\begin{array}{l}\text { Soleus } \\
\text { IMCL }\end{array}$ & $\begin{array}{l}\text { Soleus } \\
\text { EMCL }\end{array}$ & $\begin{array}{c}\text { Tibialis anterior } \\
\text { IMCL }\end{array}$ & $\begin{array}{c}\text { Tibialis anterior } \\
\text { EMCL }\end{array}$ \\
\hline BMI & $\begin{array}{c}0.11 \\
(0.71)\end{array}$ & $\begin{array}{l}-0.005 \\
(0.99)\end{array}$ & $\begin{array}{c}0.25 \\
(0.41)\end{array}$ & $\begin{array}{l}-0.05 \\
(0.87)\end{array}$ & $\begin{array}{c}0.59 \\
(0.01)\end{array}$ & $\begin{array}{c}0.37 \\
(0.15)\end{array}$ & $\begin{array}{c}0.47 \\
(0.07)\end{array}$ & $\begin{array}{c}0.44 \\
(0.08)\end{array}$ \\
\hline $\begin{array}{c}\text { Total body fat } \\
\text { mass }\end{array}$ & $\begin{array}{c}0.26 \\
(0.37)\end{array}$ & $\begin{array}{c}0.23 \\
(0.42)\end{array}$ & $\begin{array}{c}0.39 \\
(0.19)\end{array}$ & $\begin{array}{c}0.20 \\
(0.51)\end{array}$ & $\begin{array}{c}0.62 \\
(0.01)\end{array}$ & $\begin{array}{c}0.57 \\
(0.02)\end{array}$ & $\begin{array}{c}0.51 \\
(0.04)\end{array}$ & $\begin{array}{c}0.51 \\
(0.04)\end{array}$ \\
\hline $\begin{array}{l}\text { Right Leg fat } \\
\text { mass }\end{array}$ & $\begin{array}{c}0.22 \\
(0.45)\end{array}$ & $\begin{array}{c}0.32 \\
(0.26)\end{array}$ & $\begin{array}{c}0.26 \\
(0.39)\end{array}$ & $\begin{array}{c}0.39 \\
(0.18)\end{array}$ & $\begin{array}{c}0.40 \\
(0.12)\end{array}$ & $\begin{array}{c}0.49 \\
(0.05)\end{array}$ & $\begin{array}{c}0.53 \\
(0.03)\end{array}$ & $\begin{array}{c}0.56 \\
(0.02)\end{array}$ \\
\hline $\begin{array}{l}\text { Fasting } \\
\text { glucose }\end{array}$ & $\begin{array}{l}-0.04 \\
(0.90)\end{array}$ & $\begin{array}{c}0.26 \\
(0.37)\end{array}$ & $\begin{array}{l}-0.11 \\
(0.72)\end{array}$ & $\begin{array}{c}0.04 \\
(0.90)\end{array}$ & $\begin{array}{c}0.09 \\
(0.74)\end{array}$ & $\begin{array}{c}0.26 \\
(0.34)\end{array}$ & $\begin{array}{c}0.20 \\
(0.45)\end{array}$ & $\begin{array}{l}-0.08 \\
(0.77)\end{array}$ \\
\hline
\end{tabular}

IMCL $=$ intramyocellular lipid; EMCL $=$ extramyocellular lipid.

The two ethnic groups did not differ with respect to demographic variables, body composition, or muscle lipid content. On average, $\mathrm{Si}$ in the total group was $47 \%$ lower in AA $v s$. EA $(P<0.05)$. The dietary intervention subgroup population was comprised of 12 men (6 AA, 6 EA), mean age was $30.1( \pm 5.8)$ years, mean weight was $90.0( \pm 9.9) \mathrm{kg}$,

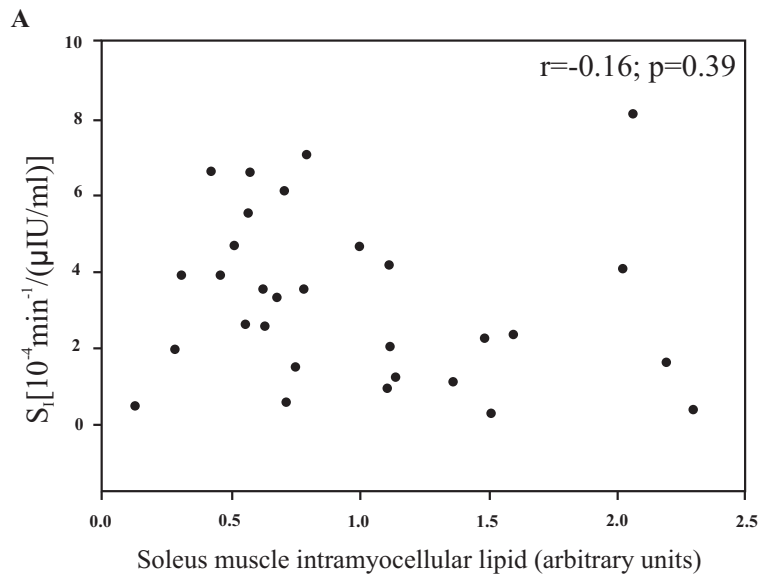

and mean BMI was $27.9( \pm 2.9) \mathrm{kg} / \mathrm{m}^{2}$. Mean total body fat mass was $25.3( \pm 6.4) \mathrm{kg}$, with a mean trunk fat mass of 13.3 $( \pm 3.6) \mathrm{kg}$, and a mean total leg fat mass of $9.0( \pm 2.5) \mathrm{kg}$.

Muscle lipids were shown to be correlated with multiple anthropometric and body composition measures in EA men but not AA men (Table 2). Muscle lipids were not associated

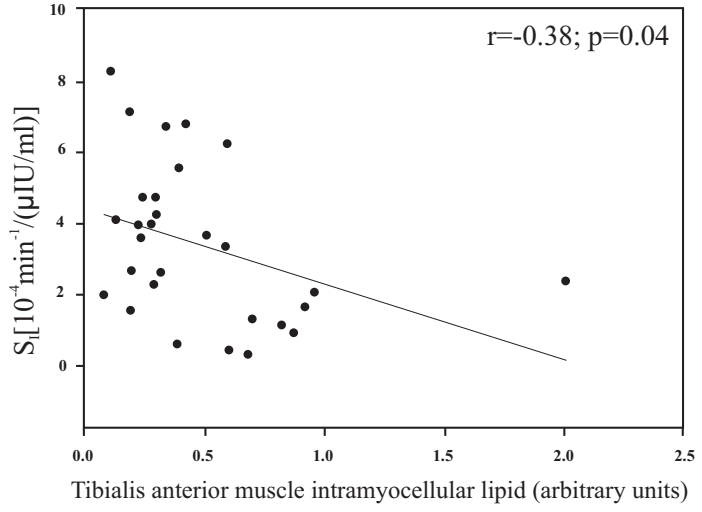

Fig. (1). A and B. Relationship between intramyocellular lipid of the soleus $(n=30)$ and the tibialis anterior $(n=29)$ muscles and insulin sensitivity $(\mathrm{Si})$ in a population of healthy, adult males.
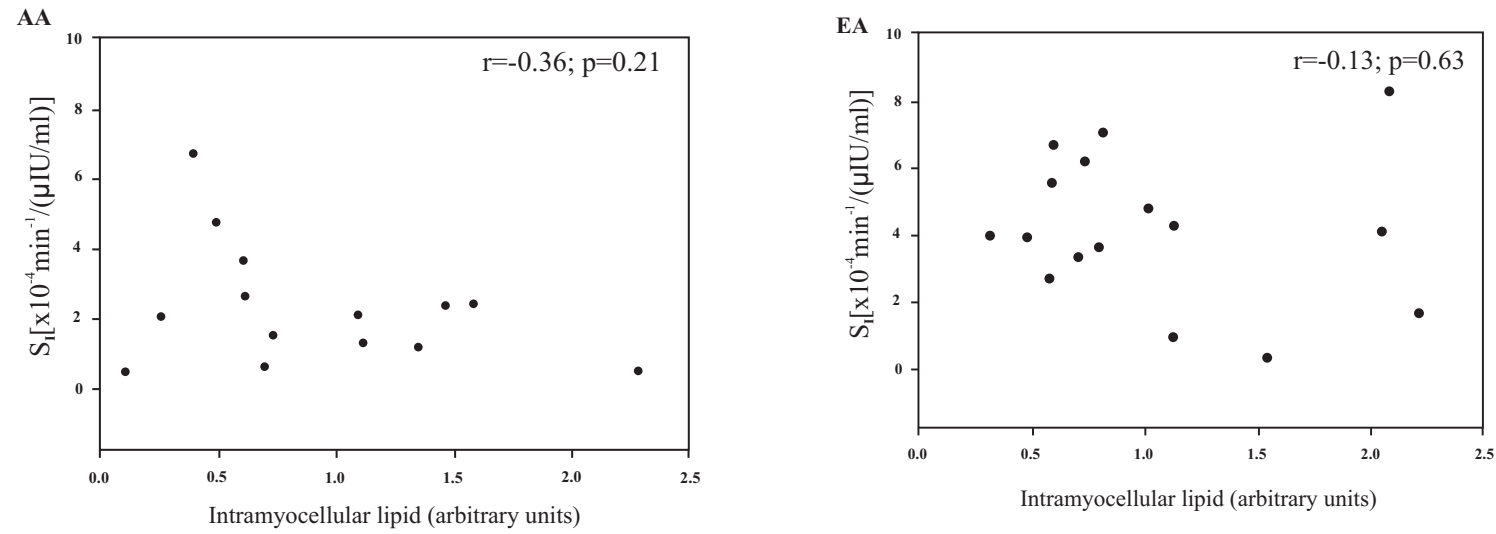

Fig. (2). Relationship of soleus muscle intramyocellular lipid to insulin sensitivity (Si) in African-American (AA) and European-American (EA) males. 

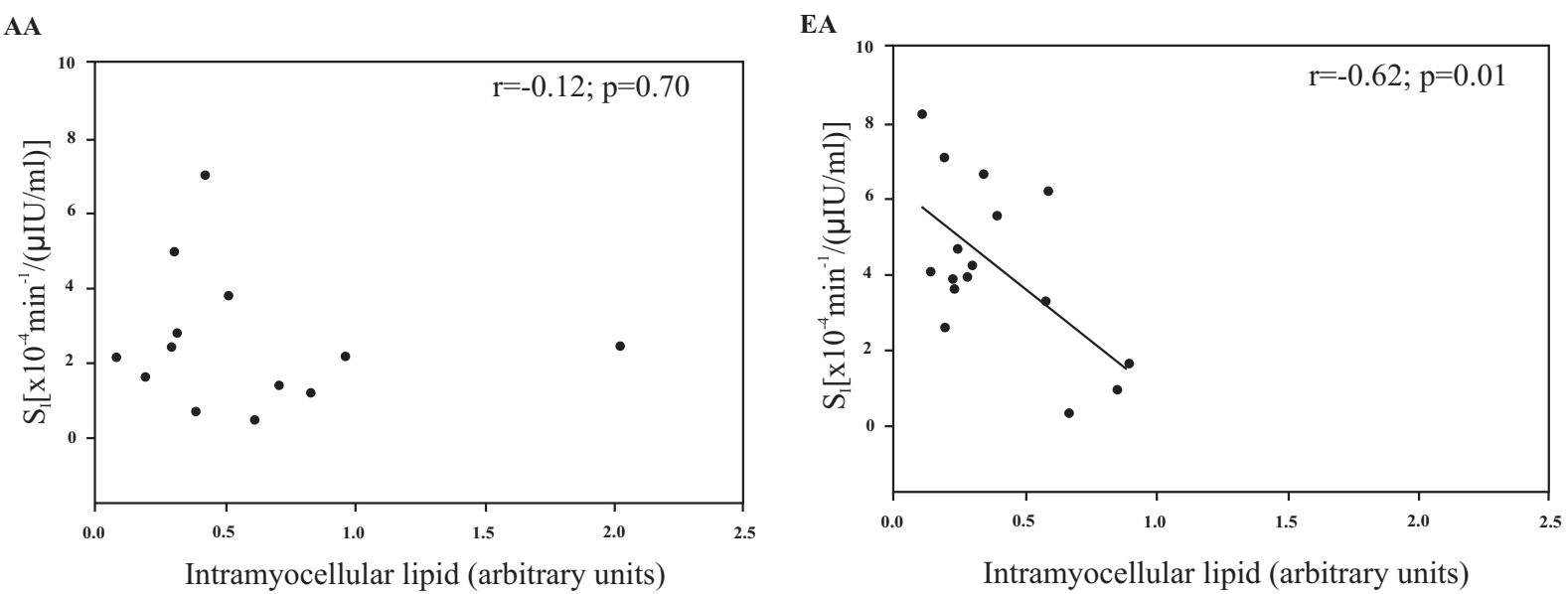

Fig. (3). Relationship of tibialis anterior muscle intramyocellular lipid to insulin sensitivity (Si) in African-American (AA) and EuropeanAmerican (EA) males.

with serum fasting glucose or insulin levels in either ethnicity.

IMCL was 2-fold higher in the soleus than in the tibialis anterior. EMCL levels between the two muscles did not differ. No significant relationships between soleus IMCL and $\mathrm{Si}$ were observed either in the entire group (Fig. 1A), or within the ethnic subgroups (Fig. 2). A significant, inverse relationship between tibialis anterior IMCL and $\mathrm{Si}$ was observed both in the entire group (Fig. 1B), and within EA subjects (Fig. 3; $P<0.05$ ). EMCL of the soleus muscle and $\mathrm{Si}$ were not associated in the total group, or in either ethnic group. The association between EMCL of the tibialis anterior and $\mathrm{Si}$ in the entire group was $r=-0.33(P=0.08)$. When examined by ethnicity, this was significant in EA ( $r=$ $-0.51, P<0.05)$, but not AA $(r=-0.37, P=0.21)$.
Soleus IMCL was reduced by $25.3 \%$ with the low-fat diet intervention $(P<0.01$; Fig. 4); no change was observed in tibialis anterior IMCL $(P=0.14$; data not shown). An increase in soleus EMCL approached significance $(P=0.07)$ but no significant change in EMCL of the tibialis anterior was observed $(P=0.11)$. Si did not change with the low-fat diet intervention ( $P=0.33$; Fig. 4).

\section{DISCUSSION}

This study was designed to test the use of a novel, fast, MRSI technique to explore the relationship of muscle lipids to insulin sensitivity in muscles of differing oxidative capacity. We found that, in this free-living population of healthy men, insulin sensitivity was correlated with tibialis anterior,

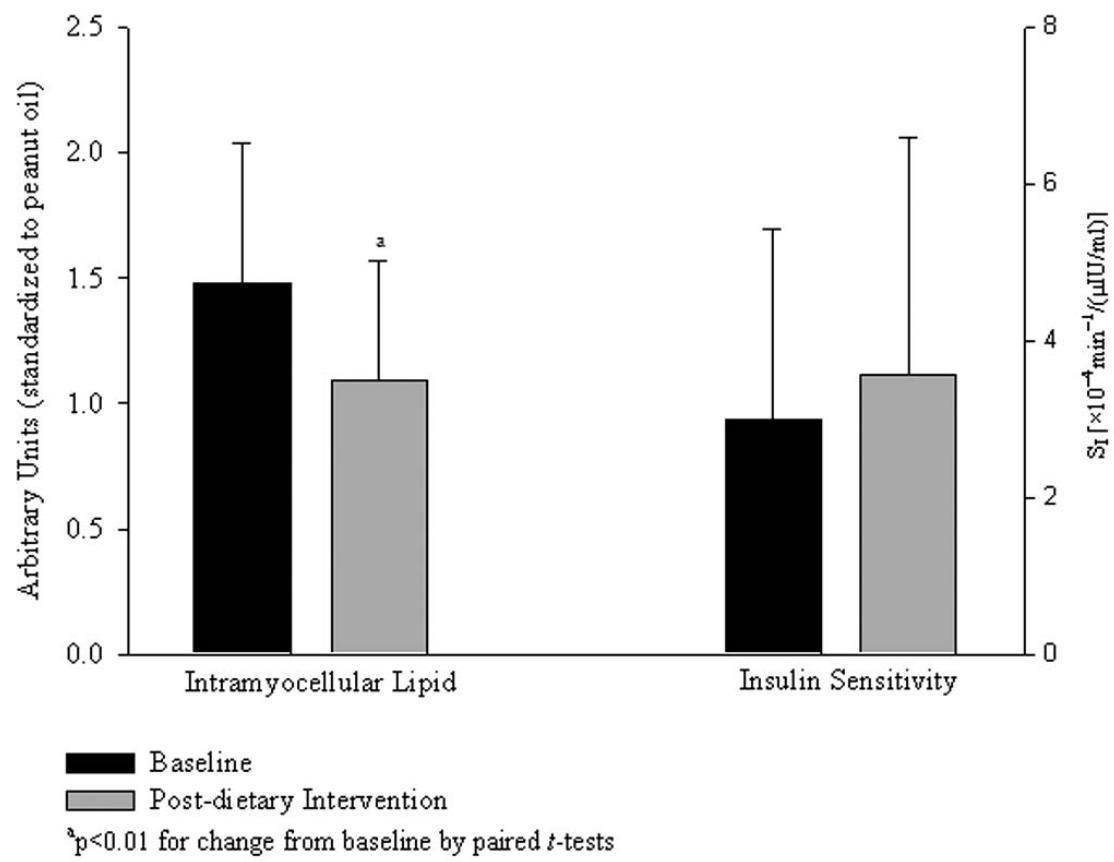

Fig. (4). Change in intramyocellular lipid of the soleus muscle and insulin sensitivity following low-fat, eucaloric diet intervention (mean \pm $\mathrm{SD})$. 
but not soleus, IMCL and that this association was apparent among EA, but not AA, subjects. Diet-induced depletion of IMCL was not associated with a change in insulin sensitivity. Results suggest that examination of multiple muscle groups with varying oxidative capacities is important in studies of muscle lipids and metabolic outcomes. Furthermore, IMCL may not be a primary determinant of insulin sensitivity among healthy young subjects, and does not appear to explain lower insulin sensitivity among AA vs. EA in this study cohort.

In the present study, we observed an association between IMCL of the tibialis anterior and $\mathrm{Si}$, but did not find a similar association between soleus IMCL and Si. Prior reports regarding the relationship between IMCL and insulin sensitivity have produced discrepant results. Significant associations have been consistently observed in obese populations [33], and in populations with, or at increased risk for, type 2 diabetes [3-5]. However, the relationship between IMCL and insulin sensitivity is less consistent in heterogenous populations of healthy adults $([5,9]$ suggesting that the relationship may be too subtle to detect, or even nonexistent, in these groups.

An alternative explanation for discrepant results in the association between IMCL and insulin sensitivity may lie with the specific muscle analyzed. Previous studies often assessed IMCL in only one muscle, and most studies used the soleus muscle. However, differential relationships of insulin sensitivity with differing muscles with varying oxidative activity have been reported in both animal and human studies. A study investigating the association of IMCL and insulin sensitivity in rats showed that the relationship differed depending on the specific muscle examined [34]. Specifically, they showed that an inverse association of glucose uptake was associated with tibialis anterior IMCL, but not soleus IMCL. Conversely, in a study of normal-toobese, nondiabetic men, Ghan and colleagues reported an inverse relationship of insulin-stimulated glucose disposal with soleus IMCL $(P=0.0049)$, but not IMCL of the tibialis anterior [35]. In that population, when subdivided into normal/overweight $v s$. more overweight groups, the relationship of soleus IMCL and insulin-stimulated glucose disposal was only apparent in the lower-weight cohort.

The mechanisms for differences among studies and populations in the IMCL-insulin sensitivity relationship are unclear. It has been suggested that the fatty acid metabolites implicated in diminished translocation and activity of cellular glucose transporters resulting in decreased insulin sensitivity may differ in content and/or action between the muscle fiber types [34]. These metabolites, such as long-chain acyl coenzyme A, diacylglycerol, and/or ceramide, may be stronger determinants of peripheral insulin resistance than are the absolute IMCL levels [6,35]. Further research is warranted to determine if the muscle-specific association between IMCL and insulin sensitivity observed in this study is due to such metabolic differences at the cellular level. The identification of differences in the relationship between IMCL of muscles of differing oxidative activity and insulin sensitivity reported by our research group and others emphasizes the importance of assessing multiple muscle groups in future clinical studies.
A secondary aim of this study was to examine ethnic differences in the relationship of muscle lipids to insulin sensitivity. In our population, insulin sensitivity was $47 \%$ lower among AA $v s$. EA, despite having no significant differences between ethnicities in body composition, fat distribution, or muscle lipid levels. These results suggest that lower insulin sensitivity among AA may not be explained by greater skeletal muscle lipid levels. However, in this population, the relationship of muscle lipids to insulin sensitivity differed with ethnicity, such that tibialis anterior IMCL and EMCL were associated with insulin sensitivity only among EA. Other studies have noted ethnic differences in IMCL-insulin sensitivity relationships. Forouhi et al. studied of a group of 40 Asian and European adult men who were matched for age and BMI [36]. In that cohort, IMCL was inversely associated with insulin sensitivity in the European men $(P=0.02)$, but not in the Asian men. Similar to our cohort of AA and EA men, their group also reported a significant ethnic difference in insulin sensitivity, such that insulin sensitivity was lower among the Asian men compared to the European men $(P=0.01)$. Unfortunately, EMCL data were not reported in that study. Our finding that associations of EMCL and insulin sensitivity differ with ethnicity is, to our knowledge, novel and should be investigated further. Additionally, research in a larger and more heterogeneous population is warranted to identify the ethnic differences in the relationship of muscle lipids to insulin sensitivity and, specifically, determine if muscle lipids play a stronger role in metabolism among EA vs. AA individuals.

A final aim of this study was to experimentally manipulate muscle lipids through diet in a small subset of these subjects and assess the effect of this manipulation on insulin sensitivity. Previous studies have shown that short-term elevations of serum lipid, via either consumption of a high-fat diet or intravenous lipid infusion, have resulted in increased IMCL with a concomitant decrease in insulin sensitivity [37]. Other studies involving low-fat diets have shown that lipid restriction following a bout of exercise, using either $10 \%$ or $15 \%$ fat eucaloric diets, impaired the replenishment of IMCL in the soleus muscle [26,38]. Based on those findings, we hypothesized that reducing the dietary fat content of a eucaloric diet to $<10 \%$ of kcals from fat would reduce IMCL and produce a corresponding increase in insulin sensitivity.

In response to the dietary intervention, soleus IMCL was significantly reduced by approximately $25 \%$. In contrast, tibialis anterior IMCL did not change. This muscle-specific response is likely related to the differing enzymatic oxidative activity between the two muscle groups [11]. During nonfasting conditions, the highly oxidative soleus muscle utilizes a higher proportion of lipid for fuel as compared to the more glycolytic tibialis anterior muscle $[39,40]$. Therefore, when lipid stores are not being replenished through diet, soleus is likely to display more severe lipid depletion. Interestingly, the diet intervention also resulted in an increase in soleus muscle EMCL that approached significance $(P=0.07)$, but not in EMCL of the tibialis anterior. It is not clear why a low-fat diet would increase EMCL. Few published studies have included EMCL data, and the metabolic relevance of EMCL is not clear. We hypothesize that increased EMCL in 
the soleus, a muscle group that relies primarily on lipid for energy, suggests that EMCL may be an intermediary storage depot for lipid being shuttled to the myocyte for energy use. To our knowledge, the finding that EMCL may change with a eucaloric, low fat diet intervention is novel, and suggests that EMCL may be relatively dynamic.

We hypothesized that reducing the dietary fat content to $<10 \%$ of kcals in a eucaloric diet would reduce IMCL and improve insulin sensitivity. Despite a significant reduction in soleus IMCL with the dietary intervention, there was no significant change in insulin sensitivity in our population of overweight but otherwise healthy men. This observation is in contrast to several studies indicating that a reduction in IMCL through exercise or calorie restriction corresponds with significant improvement in insulin sensitivity [33, 41, 42]. For example, one study of obese nondiabetic and diabetic adults provided a very-low calorie diet produced a significant decrease in IMCL $(P<0.05)$ and increase in insulin sensitivity $(P<0.01)$ after only 6 days of dietary intervention [33]. An important distinction between our study and the studies previously mentioned that may explain conflicting results is that our population was not in negative energy balance. Therefore, the process of negative energy balance itself may lead to improvements in insulin sensitivity independent of changes in IMCL. The present study is the first to show that, in a population of healthy, sedentary men, dietary fat restriction resulted in a decrease in IMCL in the absence of exercise or calorie restriction. Our study also suggests the possibility that the improvement in insulin sensitivity associated with the reduction of IMCL via caloric restriction may not be wholly due to the change in IMCL.

\section{CONCLUSIONS}

Results from this study of healthy, free-living male subjects show that this novel 2D MRSI sequence can effectively assess skeletal muscle lipid content in physiologic studies involving metabolic outcome. This technique may be useful for future studies regarding the cause-and-effect relationship between muscle lipids and metabolic health. Additionally, we have shown that the association between muscle lipids (as IMCL and EMCL) and insulin sensitivity may be influenced by both the muscle group studied and the ethnicity of the study subjects. Furthermore, significant reduction of IMCL via low-fat, eucaloric diet was not associated with a change in insulin sensitivity in this population of healthy, young males, suggesting that IMCL may not be a major determinant of insulin sensitivity in this cohort.

\section{ACKNOWLEDGEMENTS}

This work was supported by grants from the National Institutes of Health (RO1-DK067538-01A1, P30-DK-56336, 5P41RR007723, DK-38764, PO1-HL-55782), the UAB General Clinical Research Center (M01-RR-00032), the Merit Review program of the Department of Veterans Affairs, the Department of Energy (DE-FG02-01ER63119 and 1S10RR0112943), and the UAB Diabetes Research and Training Center (P60DK079626). We would like to thank the volunteers for their participation in this study.

\section{ABBREVIATIONS}

IMCL $=$ Intramyocellular lipid
EMCL $=$ Extramyocellular lipid
AA $=$ African American
EA $=$ European American
MRSI $=$ Magnetic resonance spectroscopic imaging
DXA $=$ Dual-energy X-ray absorptiometry
ROI $=$ Region-of-interest

\section{REFERENCES}

[1] Krssak M, Petersen KF, Dresner A, et al. Intramyocellular lipid concentrations are correlated with insulin sensitivity in humans: a 1H NMR spectroscopy study. Diabetologia 1999; 42: 113-16.

[2] Perseghin G, Scifo P, De Cobelli F, et al. Intramyocellular triglyceride content is a determinant of in vivo insulin resistance in humans. Diabetes 1999; 48: 1600-06.

[3] Pan DA, Lillioja S, Kriketos AD, et al. Skeletal muscle triglyceride levels are inversely related to insulin levels. Diabetes 1997; 46: 983-88.

[4] Anderwald C, Bernroider E, Krssak M, et al. Effects of insulin treatment in type 2 diabetic patients on intracellular lipid content in liver and skeletal muscle. Diabetes 2002; 51: 3025-32.

[5] Furler SM, Poynten AM, Kriketos AD, et al. Independent influences of central fat and skeletal muscle lipids on insulin sensitivity. Obes Res 2001; 9(9): 535-43.

[6] Shulman GI. Cellular mechanism of insulin resistance. J Clin Invest 2000; 6(2): 171-76.

[7] Petersen KF, Shulman GI. Pathogenesis of skeletal muscle insulin resistance in type 2 diabetes mellitus. Am J Cardiol 2002; 90(S): $11 \mathrm{G}-18 \mathrm{G}$.

[8] Garvey WT, Maianu L, Zhu JH, Brechtel-Hook G, Walace P, Baron AD. Evidence for defects in the trafficking and translocation of GLUT4 glucose ransporters in skeletal muscle as a cause of human insulin resistance. J Clin Invest 1998; 101(11): 2377-86.

[9] Phillips DIW, Caddy S, Ilic V, et al. Intramuscular triglyceride and muscle insulin sensitivity: evidence for a relationship in nondiabetic subjects. Metabolism 1996; 45(8): 947-50.

[10] Hwang JH, Stein DT, Barzilai N, et al. Increased intrahepatic triglyceride is associated with peripheral insulin resistance: in vivo MR imaging and spectroscopy studies. Am J Physiol Endocrinol Metab 2007; 293(6): E1663-69.

[11] Gregory CM, Vandenborne K, Dudley GA. Metabolic enzymes and phenotypic expression among human locomotor muscles. Muscle Nerve 2001; 24: 387-93.

[12] Megeney LA, Neufer PD, Dohm GL, et al. Effects of muscle activity and fiber composition on glucose transport and GLUT4. Am J Physiol Endocrinol Metab 1993; 264: E583-93.

[13] Dyck DJ, Peters SJ, Glatz J, et al. Functional differences in lipid metabolism in resting skeletal muscle of various fiber types. Am J Physiol Endocrinol Metab 1997; 272: E340-51.

[14] Osei K, Schuster DP. Effects of race and ethnicity on insulin sensitivity, blood pressure, and heart rate in three ethnic populations: comparative studies in African-Americans, African immigrants (Ghanaians), and White Americans using ambulatory blood pressure monitoring. Am J Hypertens 1996; 9: 1157-64.

[15] Lovejoy JC, Smith SR, Rood JC. Comparison of regional fat distribution and health risk factors in middle-aged white and African American women: the Healthy Transitions Study. Obes Res 2001; 9(1): 10-16.

[16] Gower BA, Nagy TR, Goran MI. Visceral fat, insulin sensitivity, and lipids in prepubertal children. Diabetes 1999; 48: 1515-21.

[17] Buthelezi EP, van der Merwe MT, Lonnroth PN, Gray P, Crowther NJ. Ethnic differences in the responsiveness of adipocyte lipolytic activity to insulin. Obes Res 2000; 8(2): 171-78.

[18] Ryan AS, Nicklas BJ, Berman DM. Racial differences in insulin resistance and mid-thigh fat deposition in postmenopausal women. Obes Res 2002; 10(5): 336-34. 
[19] Gallagher D, Kuznia P, Heshka S, et al. Adipose tissue in muscle: a novel depot similar in size to visceral adipose tissue. Am J Clin Nutr 2005; 81: 903-10.

[20] Albu JB, Kovera AJ, Allen L, et al. Independent association of insulin resistance with larger amounts of intermuscular adipose tissue and a greater acute insulin response to glucose in African American than in white nondiabetic women. Am J Clin Nutr 2005; 82: $1210-17$

[21] Lawrence JC, Newcomer BR, Hunter GR, et al. Abstract: Intramyocellular lipids and insulin sensitivity in premenopausal black and white women. Obes Res 2004; 12S: A145.

[22] Torriani M, Grinspoon S. Racial differences in fat distribution: the importance of intermuscular fat. Am J Clin Nutr 2005; 81(4): 73132.

[23] Newcomer BR, Lawrence JC, Buchthal S, den Hollander JA. Highresolution chemical shift imaging for the assessment of intramuscular lipids. Magn Reson Med 2007; 57(5): 848-58.

[24] Marshall WA, Tanner JM. Variations in the pattern of pubertal changes in boys. Arch Dis Child 1970; 45(239): 13-23.

[25] Naressi A, Couturier C, Devos JM, et al. Java-based graphical user interface for the MRUI quantitation package. MAGMA 2001; 12: $141-52$.

[26] Larson-Meyer DE, Newcomer BR, Hunter GR. Influence of endurance running and recovery diet on intramyocellular lipid content in women: a 1H-NMR study. Am J Physiol Endocrinol Metab 2002; 282: E95-106.

[27] Rico-Sanz J, Thomas EL, Jenkinson G, Mierisova S, Iles R, Bell JD. Diversity in levels of intracellular total creatine and triglycerides in human skeletal muscles observed by ${ }^{1} \mathrm{H}-\mathrm{MRS}$. J Appl Physiol 1999; 87: 2068 -72.

[28] Cree MG, Newcomer BR, Katsanos CS, et al. Intramuscular and liver triglycerides are increased in the elderly. J Clin Endocrinol Metab 2004; 89(8): 3864-71.

[29] Larson-Meyer DE, Smith SR, Heilbronn LK, Kelley DE, Ravussin E, Newcomer BR, Look AHEAD Adipose Research Group. Muscle-associated triglyceride measured by computed tomography and magnetic resonance spectroscopy. Obesity (Silver Spring) 2006; 14(1): 73-87.

[30] Perseghin G, Scifo P, De Cobelli F, et al. Intramyocellular triglyceride content is a determinant of in vivo insulin resistance in humans: a $1 \mathrm{H}-13 \mathrm{C}$ nuclear magnetic resonance spectroscopy assessment in offspring of type 2 diabetic parents. Diabetes 1999; 48(8): 1600-06.
[31] Bergman R, Phillips L, Cobelli C. Physiologic evaluation of factors controlling glucose tolerance in man. J Clin Invest 1981; 68: 145667.

[32] Douglas CC, Gower BA, Darnell BE, Ovalle F, Oster RA, Azziz R. Role of diet in the treatment of polycystic ovary syndrome. Fertil Steril 2006; 85(3): 679-88.

[33] Lara-Castro C, Newcomer BR, Rowell J, et al. Effects of shortterm very low-calorie diet on intramyocellular lipid and insulin sensitivity in nondiabetic and type 2 diabetic subjects. Metabolism 2008; 57(1): 1-8

[34] Korach-André M, Gounarides J, Deacon R, et al. Age and muscletype modulated role of intramyocellular lipids in the progression of insulin resistance in nondiabetic Zucker rats. Metabolism 2005; 54(4): 522-28

[35] Gan SK, Kriketos AD, Poynten AM, et al. Insulin action, regional fat, and myocyte lipid: altered relationships with increased adiposity. Obes Res 2003; 11(11): 1295-1305.

[36] Forouhi NG, Jenkinson G, Thomas EL, et al. Relation of triglyceride stores in skeletal muscle cells to central obesity and insulin sensitivity in European and South Asian men. Diabetologia 1999; 42(8): 932-35.

[37] Bachmann OP, Dahl DB, Brechtel K, et al. Effects of intravenous and dietary lipid challenge on intramyocellular lipid content and the relation with insulin sensitivity in humans. Diabetes 2001; 50: 2579-84.

[38] Decombaz J, Schmitt B, Ith M, et al. Postexercise fat intake repletes intramyocellular lipids but no faster in trained than in sedentary subjects. Am J Physiol Regul Integr Comp Physiol 2001; 218: R760-69.

[39] Kelley DE, Goodpaster B, Wing RR, Simoneau JA. Skeletal muscle fatty acid metabolism in association with insulin resistance, obesity, and weight loss. Am J Physiol 1999; 277: E1130- 41.

[40] Budohoski L, Gorski K, Nazar H, Kaciuba-Uscilko H, Terjung RL. Triacylglycerol synthesis in the different skeletal muscle fiber sections of the rat. Am J Physiol 1996; 271: E574- 81.

[41] Soloman TPJ, Sistrun SN, Krishnan RK, et al. Exercise and diet enhance fat oxidation and reduce insulin resistance in older obese adults. J Appl Physiol 2008; 104: 1313-19.

[42] Greco AV, Mingrone G, Giancaterini A, et al. Insulin resistance in morbid obesity: reversal with intramyocellular fat depletion. Diabetes 2002; 51(1): 144-51.

(C) Lawrence et al.; Licensee Bentham Open.

This is an open access article licensed under the terms of the Creative Commons Attribution Non-Commercial License (http: //creativecommons.org/licenses/by$\mathrm{nc} / 3.0 /$ ), which permits unrestricted, non-commercial use, distribution and reproduction in any medium, provided the work is properly cited. 